\title{
INTEGRATED PROCESS TARGETING AND PRODUCT UNIFORMITY MODEL FOR THREE-CLASS SCREENING
}

\author{
S. O. Duffuaa \\ \& \\ Atiq Waliullah Siddiqui \\ Department of systems Engineering, King Fahd university of Petroleum \\ \& Minerals, Dhahran, 31261, Saudi Arabia.
}

\begin{abstract}
In this paper a process targeting model for a three class screening problem is developed. The model developed, extends the work in the literature by incorporating product uniformity. The product uniformity is introduced via a Taguchi type quadratic loss function. Two cases for the process targeting are considered. In addition, an illustrative example is presented. Sensitivity analysis is also conducted to study the effect of model parameters on expected profit and optimal process mean.
\end{abstract}

\section{INTRODUCTION}

In a manufacturing environment, a product has to go across a number of processes, undergoing diverse operations before obtaining a final form. D ue to the natural and technological inconsistencies, especially systems of mechanical, chemical etc. in nature, it is bound to have some variations in the final product. In order to minimize this variation, and to improve the overall characteristics of the product, quality control became an essential part of manufacturing. Process Targeting is one of the areas in economics of quality control, which has received a lot of interest from researchers in the recent times.

The Targeting problem was initiated by Springer (1951) for the caning problem. The objective was to minimize the expected cost. Kartha (1977) presented a similar model, for the case of maximization of profit, where under filled cans are sold in a secondary market. Bisgaard $\boldsymbol{e}$ al (1984) extended the above case where the under filled cans are sold at a price proportional to the can fill. In Golhar (1987), the model presented is for the case where the under filled 
cans are reprocessed at a fixed cost. Golhar $\notin \mathrm{al}$ in (1988) considered the case where the fill is expensive and an artificial upper limit is determined alongside the process mean. Golhar et al (1992) studied the effect of variance reduction on the profit. Arcelus (1996) introduced the product uniformity via a Taguchi quadratic loss function. In Min \& al. (1997) a situation where inspection is based on three-class screening is considered.

There are many other directions in which the targeting problem has evolved e.g., use of various sampling plans instead of full inspection in Carlsson (1989), Boucher \& al (1991) and Sultan \& al. (1994) etc. O thers have considered problems having production processes with linear drift e.g., Rahim et al (1988), Sultan \& al. (1997). There are many other directions in which the work has been done and a detailed review of all of these papers is out of scope of this paper.

The paper is organized as follows. In Section 2, the model developed in this paper is described followed by two special cases in Section 3. In Section 4, an illustrative example is presented. Some results form the sensitivity analysis is discussed in Section 5. Finally, extensions and conclusions are outlined in Section 6.

\section{THE MODEL}

In this section, the model developed for targeting with product uniformity under three-class screening is presented.

Consider a production process, where the item is being sold in two different markets, having different price structures. e.g., can or bag filling, or composition of a material in a chemical solution etc. Hundred percent inspection is used to screen the product, which is performed by an automatic system. This system is considered unbiased and of negligible variance. The quality characteristic ' $\mathrm{Y}$ ' is assumed normally distributed with known variance $\sigma_{\mathrm{Y}}^{2}$. The specification limits are $L_{1}$ and $L_{2}$ (see figure 1). The item is considered grade 1 , if it falls above $L_{1}$ i.e., $Y \geq L_{1}$, for grade 2 , the specification limits are $L_{2} \leq Y<L_{1}$, the item will be scraped if $\mathrm{Y}<\mathrm{L}_{2}$. The production cost per item is $\mathrm{c}_{0}+\mathrm{cy}$, where $\mathrm{c}$ is the per unit cost e.g., per kg. or per c.c. etc. $c_{0}$ is the fixed cost and $c_{i}$ is considered as the per unit cost of inspection. Selling prices are $\mathrm{a}_{1}, \mathrm{a}_{2}$ and $\mathrm{r}$ for grade 1 , grade 2 and scrap respectively. 


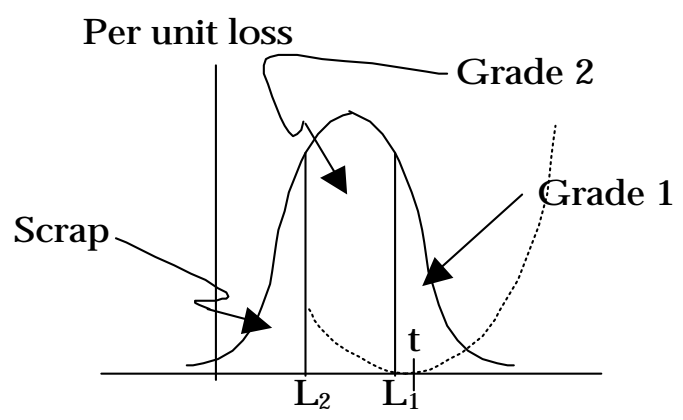

Figure 1: A production process with multi class screening \& quadratic penalty for uniformity (dotted line)

The product uniformity is introduced via a Taguchi type quadratic loss function, where $\mathrm{K}$ is the per unit per squared deviation penalty for the product being off target. The target considered is ' $t$ '. The penalty is imposed only on grade 1 and grade 2 as the scrape is not shipped to the consumer.

The profit function $\mathrm{P}(\mathrm{Y})$ can be expressed as:

$$
P(y)=\left\{\begin{array}{lc}
a_{1}-\left(c_{0}+c y+c_{i}\right)-K(y-t)^{2} & Y \geq L_{1} \\
a_{2}-\left(c_{0}+c y+c_{i}\right)-K(y-t)^{2} & L_{2} \leq Y<L_{1} \\
r-\left(c_{0}+c y+c_{i}\right) & Y<L_{2}
\end{array}\right.
$$

As can be seen from equation 1, the penalty for product inconsistency vanishes if the product falls exactly at the target ' $t$ '. The expected profit per item can be represented as:

$$
\begin{aligned}
\mathrm{E}\{\mathrm{P}(\mu, \mathrm{t})\} & =\int_{\mathrm{L}_{1}}^{\infty}\left[\mathrm{a}_{1}-\left(\mathrm{c}_{0}+\mathrm{cy}+\mathrm{c}_{\mathrm{i}}\right)-\mathrm{K}(\mathrm{y}-\mathrm{t})^{2}\right] \varphi d y \\
& +\int_{\mathrm{L}_{2}}^{\mathrm{L}_{1}}\left[\mathrm{a}_{2}-\left(\mathrm{c}_{0}+\mathrm{cy}+\mathrm{c}_{\mathrm{i}}\right)-\mathrm{K}(\mathrm{y}-\mathrm{t})^{2}\right] \varphi d y \\
& +\int_{-\infty}^{\mathrm{L}_{2}}\left[\mathrm{r}-\left(\mathrm{c}_{0}+\mathrm{cy}+\mathrm{c}_{\mathrm{i}}\right)\right] \varphi d y
\end{aligned}
$$

where $\varphi$ represents the normal distribution function in the above equation. The above equation can be represented in a simpler form as: 


$$
\begin{aligned}
\mathrm{E}\{\mathrm{P}(\mu, \mathrm{t})\}=\left[\mathrm{a}_{1} \int_{\mathrm{L}_{1}}^{\infty} \varphi d y+\mathrm{a}_{2} \int_{\mathrm{L}_{2}}^{\mathrm{L}_{1}} \varphi d y+\mathrm{r} \int_{-\infty}^{\mathrm{L}_{2}} \varphi d y\right. \\
\\
\left.\quad-\left(\mathrm{c}_{0}+\mathrm{c}_{\mathrm{i}}\right)-\mathrm{c} \mu\right]-\int_{\mathrm{L}_{2}}^{\infty}\left[\mathrm{K}(\mathrm{y}-\mathrm{t})^{2}\right] \varphi d y
\end{aligned}
$$

Letting:

$$
\begin{gathered}
\text { EPM1 }=\left[\mathrm{a}_{1} \int_{\mathrm{L}_{1}}^{\infty} \varphi d y+\mathrm{a}_{2} \int_{\mathrm{L}_{2}}^{\mathrm{L}_{1}} \varphi d y+\mathrm{r} \int_{-\infty}^{\mathrm{L}_{2}} \varphi d y\right. \\
\left.-\left(\mathrm{c}_{0}+\mathrm{c}_{\mathrm{i}}\right)-\mathrm{c \mu}\right] \\
\& \\
(y-t)^{2}=(y-\mu)^{2}+2(y-\mu)(\mu-\mathrm{t})+(\mu-\mathrm{t})^{2}
\end{gathered}
$$

equation 2 can be written as:

$$
\begin{aligned}
& E\{P(\mu, t)\}=E P M 1-K\left[\int_{L_{2}}^{\infty}(y-\mu)^{2} \varphi d y+\right. \\
& \left.2(\mu-t) \int_{L_{2}}^{\infty}(y-\mu) \varphi d y+(\mu-t)^{2} \int_{L_{2}}^{\infty} \varphi d y\right]
\end{aligned}
$$

Letting $_{\mathrm{z}}=\frac{\mathrm{y}-\mu}{\sigma_{\mathrm{y}}}$ for standard normal, the above equation can be restated as:

$$
\begin{aligned}
\mathrm{EPMB}=\mathrm{EPM} 1 & \left.-\mathrm{K}\left|\varphi\left(\Gamma_{2}\right)\right| \sigma^{2} \mathrm{z} \varphi\left(\Gamma_{2}\right)+2 \sigma(\mu-\mathrm{t})\right] \\
& \left.\left.+\left[\sigma_{\mathrm{Y}}^{2}+(\mu-\mathrm{t})^{2}\right] 1-\Phi\left(\Gamma_{2}\right)\right]\right]
\end{aligned}
$$

\section{SPECIAL CASES}

In this section, two special cases are discussed, depending upon the socially ideal target. In special case I, the target is assumed to be set at the process mean, while In the other case the target is assumed to be set at the specification limit of the grade 1. Point to note is that, the target ' $\mathrm{t}$ ' is a socially set ideal value that depends on the consumers, and it can be located any where. The above two cases are for the situations where the consumers is concerned about 'just consistent' in the first case while in second case, the consumers ideal is a specific point which is $\mathrm{L}_{1}$. 
SPECIAL CASE I: In this case the target value ' $t$ ' for the uniformity of the product is set at the mean of the process itself. The expected profit for the case I can be written as:

$$
\begin{aligned}
\mathrm{EPM} 3= & \mathrm{EPM} 1 \\
& -\mathrm{K}\left[\varphi\left(\Gamma_{2}\right)\left[\sigma_{\mathrm{y}}^{2} z \varphi\left(\Gamma_{2}\right)\right]+\sigma_{\mathrm{Y}}^{2}\left[1-\Phi\left(\Gamma_{2}\right)\right]\right]
\end{aligned}
$$

SPECIAL CASE II: In this case the target is assumed set at the specification limit of the grade I i.e., $\mathrm{L}_{1}$. The expression for the expected profit for this case II is given in equation (6):

$$
\begin{gathered}
\text { EPM3 }=\text { EPM } 1-K\left[\varphi\left(\Gamma_{2}\right)\left[\sigma^{2} \mathrm{z} \varphi\left(\Gamma_{2}\right)+2 \sigma\left(\mu-L_{1}\right)\right]\right. \\
\left.+\left[\sigma_{Y}^{2}+\left(\mu-L_{1}\right)^{2}\right]\left[1-\Phi\left(\Gamma_{2}\right)\right]\right]
\end{gathered}
$$

In special case one, since the target is set at the mean of the process itself, location of the optimal mean is not very much affected by the target. On the other hand, the effect on optimal mean, for special case II, is translated significantly and the location of the optimal mean is forced closer to the specification limit $\mathrm{L}_{1}$ as compared to special case I. This inference is further justified by the sensitivity analysis.

\section{ILLUSTRATIVE EXAMPLE}

Consider a packing plant of a cement factory. The plant consists of two processes a filling process and an inspection process. Each cement bag processed by filling machine is moved to the loading and dispatching phase on the conveyor belt. Inspection is per-formed by automatic weighing system and is assumed error free. Suppose that the cost components and the specification limits are $a_{1}=\$ 5.5, a_{2}=\$ 4.9, r=\$ 2.5, c_{0}=\$ 0.1, c_{1}=\$ 0.04, c=\$ 0.12, L_{1}=41.5 \mathrm{~kg}$, $\mathrm{L}_{2}=40.0 \mathrm{~kg}, \mathrm{k}=0.25$ and $\sigma_{\mathrm{y}}^{2}=(1.5)^{2}$.

The expected profit and the optimal values of the mean and the cut off values are found out to be:

\section{1) No uniformity penalty is assumed}

The results are the same as obtained from Min at al. (1997)

$E(p)=\$ 1.28671 /$ unit 
$\mu=44.3291 \mathrm{~kg}$

\section{2) Case I: tanget at $\mu$}

$E(p)=\$ 1.10853 /$ unit

$\mu=44.392 \mathrm{~kg}$

Consider the case where the penalty for non uniformity was neglected, the gain in expected profit will be reflected if the mean obtained from model 1 is substituted in model obtained in case I i.e.,

$E(p)=\$ 1.10635 /$ unit

Gain in profit: $\$ 0.00218$ / unit or approximately $0.197 \%$. Now considering a million units produced per annum by a manufacturer, the net gain in expected profit per year is $\$ 2180$.

\section{3) Case II: target at $\mathrm{L}_{1}$}

$\mathrm{E}(\mathrm{p})=\$ 0.90272 /$ unit

$\mu=42.9241 \mathrm{~kg}$

Consider the case where the penalty for non uniformity was neglected, the gain in expected profit will be reflected $\mathbf{f}$ the mean obtained from model 1 is substituted in the model obtained in case II i.e.,

$E(p)=\$ 0.69055 /$ unit

Gain in profit: $\$ 0.21217 /$ unit or approximately $23.50 \%$. Now considering a million units produced per annum by a manufacturer, the net gain in expected profit per year is $\$ 212170$, which, as can be seen, is a huge gain. In other words setting the mean higher for selling more products will result in a loss of $\$ 212170$ in terms of inconsistency penalties.

\section{SENSITIVITY ANALYSIS}

In this parametric analysis, conducted on the parameters of the example, the effect of different parameters on the output values i.e., the expected profit, the optimal mean are studied. There are two special cases derived in the section 3. Both of these cases have been evaluated in this sensitivity analysis. Following is a brief summary of the results. 


\section{SPECIAL CASE I}

Figure 2: $\mathrm{E}(\mathrm{p})$ versus a2-r/ a1-a2 at sigma $=1.5$

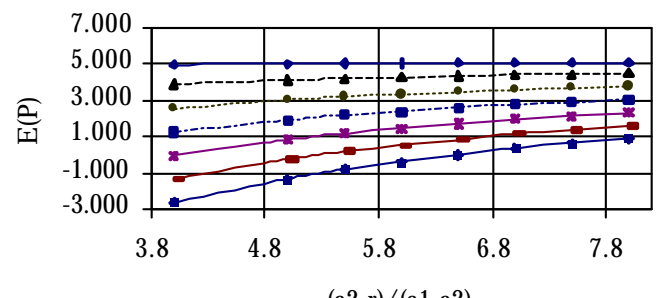

c/ (a1-a2)
Figure 3: $\mathrm{E}(\mathrm{p})$ versus $\mathrm{c} / \mathrm{a} 1-\mathrm{a} 2$ at sigma $=1.5$
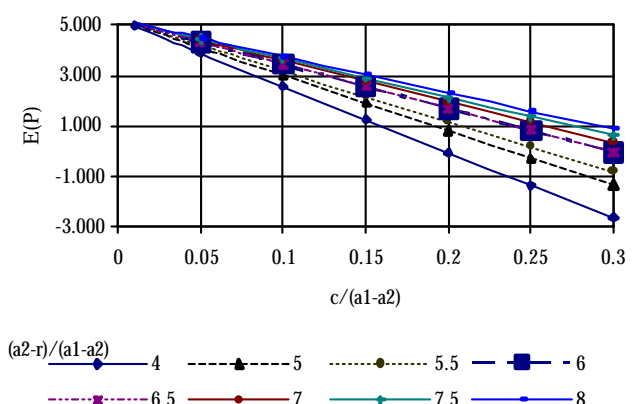

The parameters varied are mainly $\frac{c}{a_{1}-a_{2}}$ and $\frac{a_{2}-r}{a_{1}-a_{2}}$ representing the cost and the selling prices in dimensionless form and $\left(\sigma_{y}\right)^{2} . \mathrm{K}$ is taken at 0.05 . The graphs from some of the results are shown in figure $2 \& 3$ for the expected profit and figure $4 \& 5$ are the variations in the optimal mean for the same set of problems.

The results show, that the expected profit decrease sharply with the cost parameter as compared to the decrease in the selling price parameter. Similar is the case of the variation in the optimal process mean i.e., the result show a sharp decrease with cost parameter in the optimal mean as compared to the decrease in selling price parameter. This sharp decrease with increase in cost parameter is due to the fact that the cost is directly related to the mean as can be seen from equation (2). As compared to the cost parameter, selling price parameter are related to the probability of the product falling in the respective grade, (see equation (2)) therefore the effect of the cost will always going to be pronounced as compared to the selling price.

Figure 4: Optimal Mean versus a2-r/a1-a2 at sigma $=1.5$

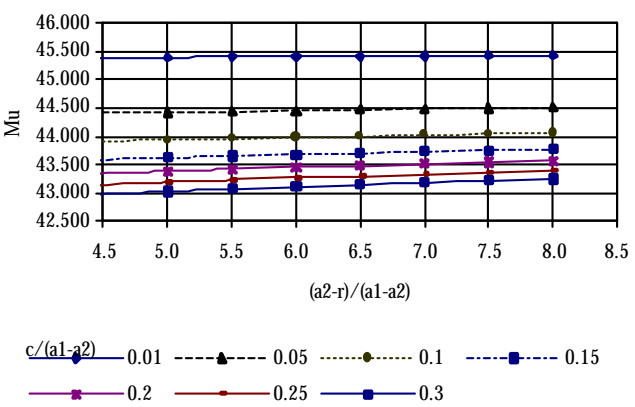

Figure 5: 0 ptimal Mean versus c/ a1-a2 at sigma $=1.5$

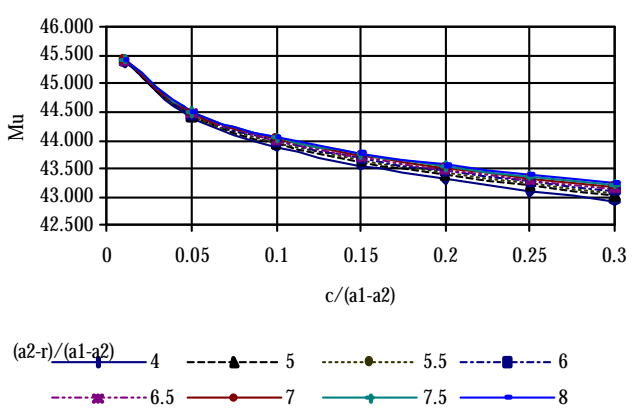




\section{SPECIAL CASE II}

Figure 6: $\mathrm{E}(\mathrm{p})$ versus a2-r/ a1-a2 at sigma $=1.5$

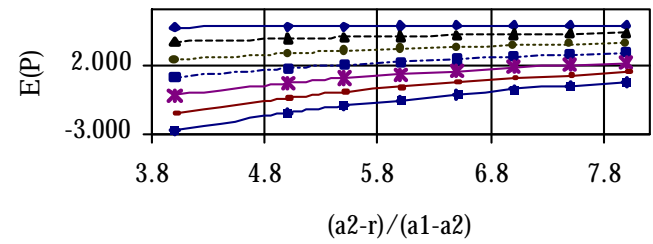

c/ (a1-a2)

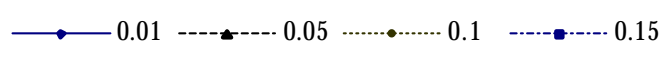

Figure 7: $\mathrm{E}(\mathrm{p})$ versus $\mathrm{c} / \mathrm{a} 1-\mathrm{a} 2$ at sigma $=1.5$

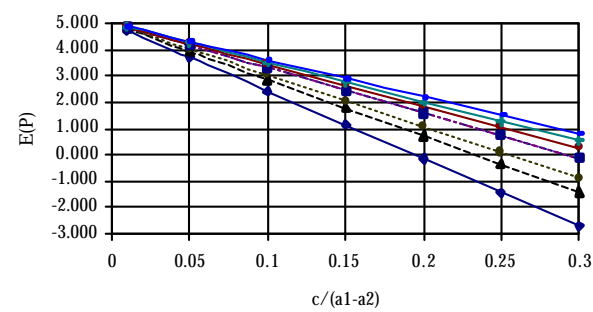

(a2-r)/ (a1-a2)

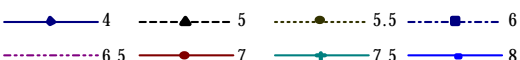

For the same set of problem the results for special case II are shown in figure $6 \& 7$ for expected profit and figure $8 \& 9$ for the optimal mean. The effect of cost and price parameters are similar in nature as in special case I, however a significant observation is the fact that the mean is forced closer to the specification limit $\mathrm{L}_{1}$ in special case II. Even though the value of $\mathrm{K}$ is set smaller, the effect on the mean is significant and consequently on the expected profit. These results from the sensitivity analysis and the illustrative example suggest that; care must be required for neglecting the penalties associated with the inconsistencies even at very low level of $\mathrm{K}$ especially for the case where the target is taken fixed (not related to the optimal mean) at a point other than the mean of the process itself.
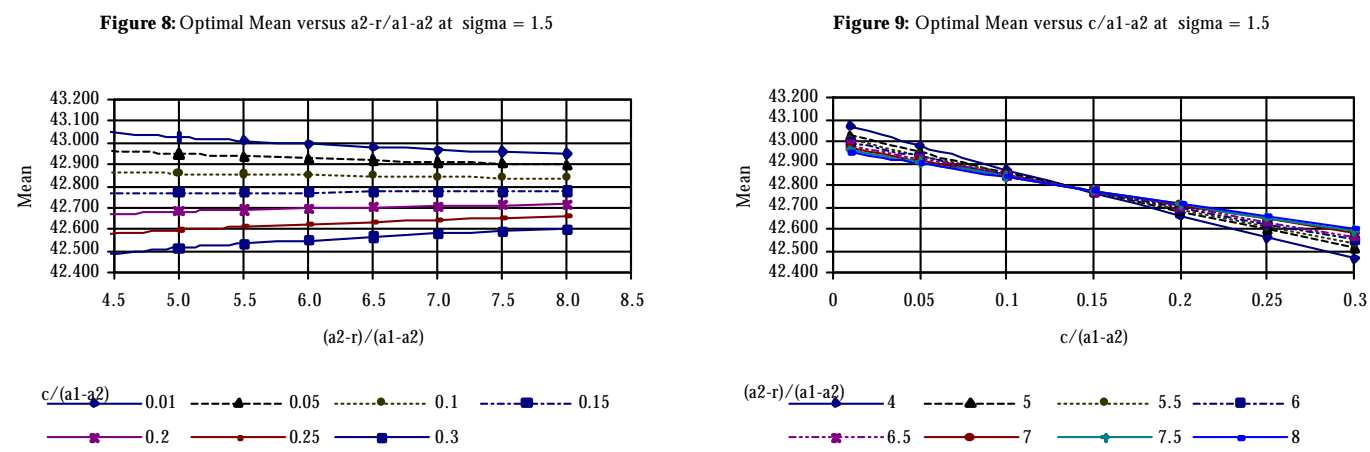

\section{CONCLUSION \& SUGGESTIONS}

In this work, the Taguchi quadratic function is integrated with the three-class screening targeting model. The model can further be modified, more in line with the concepts of quality engineering. The proposed changes are to modify the above model and apply the quadratic 
function that is asymmetric in nature in order to take care of the objectives like larger the better and smaller the better etc.

The illustrative example is section 4 suggests the importance of using this model, particularly if the target is set away from the optimal mean. The results show the loss incurred if the inconsistency penalties are neglected are quite significant in some cases.

The effect of the inconsistency parameter ' $\mathrm{K}$ ' is not studies in detail in this paper, however the effect of ' $\mathrm{K}$ ' on profit gain, if the new model is used instead of the old model, is also a worthwhile and interesting study. The model can further be extended by introducing sampling plans and systems with linear drift.

An immediate extension suggested from the current work is to generalize it for the case of multi-class screening. Although two and three-class screening are the most prevalent systems of screening, however a number of cases are found in the industry where more than threeclass screening is used.

\section{BIBLIOGRAPHY}

K. S. Al-Sultan (1994) "An Algorithm for the Determining of the Optimum Target Value for Two Machines in Series with Quality Sampling Plans". International Journal of Productions Research, Vol. 32, No. 1, 1994, 37 - 45

K. S. Al-Sultan and M. F. Pulak (1997) "Process Improvement by Variance Reduction for A Single Filling Operation with Rectifying Inspection" Production Planning and Control, Vol. 8, No. 5, 1997, 431 - 436.

F. J. Arcelus (1996) "Uniformity of the Production Vs. Conformance to Specifications in the Canning Problem". Optimization in Quality Control, 1996

S. Bisgaard, W. Hunter and L. Pallensen (1984) "Economic Selection of Quality of Manufacturing Product". Technometrics, Vol. 26, 1984, 9 - 18

T. Boucher and M. Jafari (1991) "The Optimum Target Value for Single Filling O perations with Q uality Sampling Plans". Journal of Quality Technology, Vol. 23, No. 1, 1991, 44 - 47

O. Carlsson (1989) (a) "Economic Selection of a Process Level under Acceptance Sampling Variables". Engineering Costs and Production Economics, Vol. 16, No. 2, 1989, 69 - 78 
D. Golhar (1987) "D etermining of the Best Mean Contents for a Canning Problem". Journal of Quality Technology, Vol. 19, No. 2, April 1987, 82 - 84

D. Golhar and S. Pollock (1988) "D etermining of the Optimal Process Mean and the Upper Limit for a Canning Problem”. Journal of Q uality Technology, Vol. 20, No. 3, July 1988, 188 $-1992$

D. Golhar and S. Pollock (1992) "Cost Saving Due to Variance Reduction in a Canning Problem". IIE Transactions.

W. Hunter and C. Kartha (1977) "D etermining the Most Profitable Target Value for a Production Process". Journal of Q uality Technology, Vol. 9, No. 4, Oct. 1977, 176 - 181

M. K. Lee and J. S. Jang (1997) "The Optimal Target Values for a Production Process with Three Class Screening”. International Journal of Productions Economics, Vol. 49, 1997, 91 99

C. Springer (1951) "A Method for Determining the Most Economic Position of a Process Mean”. Industrial Quality Control, Vol. 8, No. 1, July 1951, 36 - 39

M. A. Rahim and P. K. Banerjee (1988) "Optimal Production Run for a Process with Random Linear D rift”. O MEGA, Vol. 16, No. 4, 1988, 347 - 351 